\title{
Molecular variations in Th1-specific cell surface gene Tim-3
}

\author{
Soo-Cheon Chae', Ju-Hee Song ${ }^{1}$, \\ Pann Pounsambath ${ }^{1}$, Hai-Ying Yuan ${ }^{1}$, \\ Jae-Hoon Lee ${ }^{2}$, Jeong-Joong $\mathrm{Kim}^{3}$, \\ Yong-Chul Lee ${ }^{4}$ and Hun-Taeg Chung ${ }^{1,5,6}$ \\ ${ }^{1}$ Genome Research Center for Immune Disorders \\ ${ }^{2}$ Department of Otolaryngology \\ ${ }^{3}$ Department of Anatomy \\ Wonkwang University School of Medicine \\ Iksan, Chonbuk 570-749, Korea \\ ${ }^{4}$ Department of Internal Medicine \\ Research Center for Allergic Immune Diseases \\ Chonbuk National University Medical School \\ Chonju, Chonbuk 560-180, Korea \\ ${ }^{5}$ Department of Microbiology and Immunology \\ Wonkwang University School of Medicine \\ Iksan, Chonbuk 570-749, Korea \\ ${ }^{6}$ Corresponding author: Tel, 82-63-8506762; \\ Fax, 82-63-8515066, E-mail, htchung@wonkwang.ac.kr
}

\section{Accepted 28 April 2004}

Abbreviations: AHR, airway hyperreactivity; HAVCR, hepatitis A virus cellular receptor; SNP, single nucleotide polymorphism; TIM, T-cell immunoglobulin domain and mucin domain

\begin{abstract}
The family of T-cell immunoglobulin domain and mucin domain (TIM) proteins is identified to be expressed on T cells. A member of Tim family, Tim-3 (T cell immunoglobulin mucin 3 ) is selectively expressed on the surface of differentiated Th1 cells. Tim-3 might have an important role in the induction of autoimmune diseases by regulating macrophage activation and interacts with Tim-3 ligand to regulate Th1 responses. To determine the variation sites in the coding and promoter region of human Tim-3 gene, we performed variation scanning by direct sequencing using the genomic DNA isolated from the patients with asthma or allergic rhinitis and healthy controls without asthma and allergic rhinitis. We identified four single nucleotide polymorphisms (SNPs) including one novel SNPs (-1541C $>T)$ and two variation sites $\left(-1292_{-}-1289\right.$ delTAAA and $-1282-1278$ dupTAAAA) in the coding and promoter region of human Tim-3 gene in both
\end{abstract}

\author{
the patients and healthy groups.
}

Keywords: asthma; hypersensitivity; polymorphism, rhinitis; Tim-3

\section{Introduction}

Naive T helper cells differentiate into Th1 or Th2 cells by multiple factors, which include cytokine profile, type of antigen, delivery route of antigens, transcription factors and signaling pathways (O'Garra 2000; Ho and Glimcher 2002). The balance between Th1 and Th2 cells is critical in the immune response to pathogens, tumor antigens and allergens. Th1 and Th2 cells cross-regulate the differentiation of partner subset. The predominant induction of Th2 cells inhibits autoimmune diseases and the predominant induction of Th1 cells can regulate induction of asthma and allergic diseases (Lack et al., 1994; Nicholson et al., 1995; Hofstra et al., 1998).

Recently, the family of T-cell immunoglobulin domain and mucin domain (TIM) proteins was identified to be expressed on T cells (Mclntire et al., 2001). All three of this gene family encodes cell surface glycoproteins with common structural motifs, including a signal peptide, Ig domain, mucin domain, transmembrane region and intracellular tail with phosphorylation sites. The predicted structure of TIM-1 contains two tyrosine residues and includes a conserved tyrosine kinase phosphorylation motif on the cytoplasmic region. TIM-2 also has two extracellular N-linked putative glycosylation sites and an intracellular tyrosine kinase phosphorylation motif (Mclntire et al., 2001). TIM-3 (also known as HAVCR-2, hepatitis A virus cellular receptor 2) encodes the integral membrane glycoprotein with multiple extracellular putative glycosylation sites and an intercellular tyrosine phosphorylation motif. The human homologue of Tim-1, which lies at human chromosome $5 q 33.2$, encodes for the cellular receptor for hepatitis $A$ virus (Feigelstock et al., 1998). TIM-1 was considered as a membrane protein that is associated with the development of Th2 biased immune responses and may be selectively expressed on Th2 cell (Mclntire et al., 2001; Umetsu et al., 2002). More recently, Kumanogoh et al. reported that TIM-2 interacts with the class IV semaphorin Sema4A, which enhances the activation and differentiation of $T$ cells (Kumanogoh et al., 2002). TIM-3 was not expressed on naive $T$ cells, B cells, macrophages or dendritic cells. TIM-3 was only present at the highest level in Th1 cells and has an 
essential role in Th1 responses and macrophage activation (Monney et al., 2002). Comparison of the sequence of the coding regions for the three Tim genes in the two strains of mice showed sequence variants in Tim-1 and Tim-3 (Mclntire et al., 2001). The results of above studies led us to identify the polymorphism of Tim-3 genes in a Korean.

In this study, we scanned the variation sites of human Tim-3 gene using the genomic DNA isolated from 24 controls without asthma and allergic rhinitis, 24 asthma and 26 allergic rhinitis patients by direct sequencing.

\section{Materials and Methods}

\section{Subjects and DNA samples}

Blood samples were obtained from 24 unrelated asthma patients, 26 unrelated allergic rhinitis patients and 24 unrelated controls without asthma and allergic rhinitis. The asthma and allergic rhinitis patients were recruited from our outpatient clinic at Chonbuk National University Hospital and Wonkwang Medical Hospital, respectively. The healthy controls were recruited from the general population who took a comprehensive medical testing at Wonkwang University Hospital. The all subjects used in this study were Korean living in the same area. Genomic DNA was extracted from leukocytes of peripheral blood by a standard phenol-chloroform method or by Invisorb spin blood Maxi kit (Invitek, Germany) according to manufacturer's direction.

\section{PCR and sequencing analysis}

The promoter and coding regions of Tim-3 were partially amplified using the seven primer pairs (Table 1). PCR was carried out in a $25 \mu$ reaction volume containing $50 \mathrm{ng}$ of genomic DNA, $0.5 \mu \mathrm{M}$ of the primers, $0.2 \mathrm{mM}$ of dNTP, $1.5 \mathrm{mM}$ of $\mathrm{MgCl}_{2}, 10 \mathrm{mM}$ of Tris- $\mathrm{HCl}(\mathrm{pH}$ 8.3) and one unit of Ex Taq polymerase (TaKaRa). PCR was run for 30 cycles of denaturation at $95^{\circ} \mathrm{C}$ for $30 \mathrm{~s}$, annealing at melting temperature of the each primer pair for $40 \mathrm{~s}$, and extension at $72^{\circ} \mathrm{C}$ for $1 \mathrm{~min}$ or $2 \mathrm{~min}$. Both sense and antisense strands of PCR products were directly sequenced using the same primers used for the PCR amplification. Five primers (Tim-3-PF1: 5'-TTAAGAGCCTTGACCAAGTTCA-3', Tim-3-PF2; 5'-ATACAAAATTATCCGGGGTGGT-3', Tim-3-PF3; 5'-TGTGAGCTCACTTCCCTTTTAT-3', Tim-3-PF4; 5'-GAGAGCCATGCATGTATTATCT-3', and Tim-3-PF5; 5'-CATGACTGAGTAGCGTTTTCTT-3') were additionally used to sequence the promoter region. PCR products purified by PCR purification kit (Millipore) were used as template DNA for cycle sequencing. The PCR for sequencing was performed using BigDye Terminator cycle sequencing and analyzed using an $A B I 3100$ Prism Automated DNA sequencer (Applied Biosystems) by according to the manufacturer's instruction.

\section{Statistical analysis}

The polymorphisms of Tim-3 gene were identified in 74 unrelated Korean individuals by general direct sequencing method (Kim et al., 2003). The asthma or allergic rhinitis patients and healthy controls were compared using case-control association analyses. Allele carrier frequency was defined as the percentage of the individuals carrying the allele among the total number of the individuals. Fisher's exact test or $x^{2}$ test from $2 \times 3$ contingency table was applied to analyze the comparison of the frequency of discrete variables between unrelated asthma or rhinitis patients and unrelated healthy controls. $P$-value less than 0.05 were considered to indicate statistical significance. The reference sequence is based on the sequence of human chromosome 5 clones CTB120L21.

Table 1. Primer sequences for amplifying the coding and promoter regions of the Tim-3.

\begin{tabular}{|c|c|c|c|}
\hline Amplified region & Primer name & Primer sequence $\left(5 \longrightarrow 3^{\prime}\right)$ & $\begin{array}{l}\text { Product } \\
\text { size (bp) }\end{array}$ \\
\hline Promoter and Exon 1 & Tim3-1F/Tim3-1R & TTAAGAGCCTTGACCAAGTTCA/TACAATGGCCATCCTTGTATCT & 1990 \\
\hline Exon 2 & Tim3-2F/Tim3-2R & GCAGTTTTCCTGAAATGGAGTA/GAAGTCAGAGATGAGAACAATC & 491 \\
\hline Exon 3 & Tim3-3F/Tim3-3R & ССTTTGATTCCCTGAAATAAGC/TGAATTCAGAGCCAGCTAAAGA & 203 \\
\hline Exon 4 & Tim3-4F/Tim3-4R & TTGGCTATTTTTCCTCTCTCTC/GACAAGTACGGAGTAGAATTCA & 221 \\
\hline Exon 5 & Tim3-5F/Tim3-5R & GGATGGTCCAATTCACAAATCT/GAATTTGTTATCAGAGGGAGAG & 280 \\
\hline Exon 6 & Tim3-6F/Tim3-6R & GCTTCCAGTGAACCTATTTGAT/TCTAAGGCACACGTTGAGAG & 228 \\
\hline Exon 7 & Tim3-7F/Tim3-7R & TGTTACCTGGGAAATGCTTAAC/AGGCAATGACATGCCTGTTTAA & 440 \\
\hline
\end{tabular}




\section{Results and Discussion}

The genomic size of human Tim-3 is about $23 \mathrm{~kb}$ and consists of 301 amino acids, including a signal peptide, an IgV domain, a mucin domain, a transmembrane region and a cytoplasmic region. To determine the variation sites in the coding and promoter region of human Tim-3 gene, we performed variation scanning by direct sequencing using the genomic DNA isolated from 24 unrelated asthma patients, 26 unrelated allergic rhinitis patients and 24 unrelated healthy controls. We identified a total of four single nucleotide polymorphisms (SNPs), -1541C $>\mathrm{T},-1516 \mathrm{G}>\mathrm{T}$ (rs10053538), -574T $>\mathrm{G}$ (rs10515746) and 4259G $>$ $T$ (rs1036199), and two variation sites, -1292 -1289delTAAA and -1282_-1278dupTAAAA, in the coding and promoter region of human Tim-3 gene (Table 2; Figure 1).

Among the SNPs identified in this study, three SNPs, $-1541 C>T$ (position is calculated from the translation start site), $-1516 \mathrm{G}>\mathrm{T}$ (rs10053538) and $-574 T>G$ (rs10515746), were located in promoter region and one SNP, 4259G $>T$ (rs1036199), was located in exon 3. Interestingly, -1516G $>$ T (rs10053538) located at the putative transcriptional binding site of p300. T allele of the both $-1767 \mathrm{~T}>\mathrm{G}$ and $-882 \mathrm{~T}>\mathrm{C}$ located in promoter region was changed to $G$ and $C$, respectively, on the all of genomic DNA used in this study (data not shown). We found two different sequences between our sequence and NCBI reference sequence (human chromosome 5 clone CTB-120L21). The allele $T(-1767$ and -882$)$ of reference sequence was substituted to allele $G$ and $C$, respectively on the all of genomic DNA samples isolated from 74 Korean. A variation site of promoter region, 1572insC, was also newly identified on our sequence (data not shown). These results led us to think that it might be the sequencing error of the reference sequence or it may be a specific nucleotide sequence of Korean. The coding region of human Tim-3 gene was consisted of seven exons but only one SNP are identified in exon 3 (Table 2). The change of allele at $4259 \mathrm{G}>\mathrm{T}$ (rs1036199) of exon 3 is resulted inamino acid substitution; arginine to leucine. A varia-

Table 2. Genotype and allele frequencies of the Tim-3 variations between the patients with asthma or allergic rhinitis and controls.

\begin{tabular}{|c|c|c|c|c|c|c|c|}
\hline \multirow{2}{*}{ Position } & \multirow{2}{*}{ Genotype/Allele $^{a}$} & \multirow{2}{*}{ Control $(n)$} & \multirow{2}{*}{ Asthma $(n)$} & \multirow{2}{*}{ Rhinitis $(n)$} & \multirow{2}{*}{ Freq. $^{\mathrm{b}}$} & \multicolumn{2}{|l|}{$P^{\mathrm{c}}$} \\
\hline & & & & & & vs. asthma & Rhinitis \\
\hline \multirow[t]{3}{*}{$-1541 C>T^{d}$} & $\mathrm{CC}$ & 23 & 24 & 26 & 0.007 & - & - \\
\hline & CT & 1 & 0 & 0 & & & \\
\hline & $\mathrm{TT}$ & 0 & 0 & 0 & & & \\
\hline \multirow[t]{3}{*}{$-1516 \mathrm{G}>\mathrm{T} \quad(\mathrm{rs} 10053538)$} & GG & 18 & 19 & 23 & 0.095 & 0.942 & 0.465 \\
\hline & GT & 6 & 5 & 3 & & & \\
\hline & $\mathrm{TT}$ & 0 & 0 & 0 & & & \\
\hline \multirow[t]{3}{*}{$-574 T>G \quad(r s 10515746)$} & TT & 0 & 0 & 0 & 0.074 & 0.470 & 0.995 \\
\hline & TG & 2 & 5 & 4 & & & \\
\hline & GG & 22 & 19 & 22 & & & \\
\hline \multirow{3}{*}{$4259 \mathrm{G}>\mathrm{T} \quad(\mathrm{rs} 1036199)$} & GG & 0 & 0 & 0 & 0.028 & - & - \\
\hline & GT & 0 & 0 & 4 & & & \\
\hline & TT & 24 & 24 & 22 & & & \\
\hline \multirow[t]{5}{*}{$-1292 \_-1289$ delTAAA $^{d}$} & Genotype1 & 0 & 0 & 0 & 0.122 & 0.942 & 0.990 \\
\hline & Genotype2 & 6 & 5 & 7 & & & \\
\hline & Genotype3 & 18 & 19 & 19 & & & \\
\hline & Allele1 & 6 & 5 & 7 & & 0.762 & 1.000 \\
\hline & Allele2 & 42 & 43 & 45 & & & \\
\hline \multirow[t]{2}{*}{$-1282 \_-1278 d u p T A A A A^{d}$} & Allele1 & 6 & 5 & 7 & 0.122 & 0.762 & 1.000 \\
\hline & Allele2 & 42 & 43 & 45 & & & \\
\hline
\end{tabular}

${ }^{a} G$ Genotype 1, 2 and 3 mean the homozygous with reference sequence, the heterozygous by reference and variation sequence and the homozygous with variation sequence, respectively; Allele 1 and 2 mean the reference sequence and the variation sequence, respectively. ${ }^{b}$ Frequencies of rare alleles, 'Fisher's exact test or $x^{2}$ test from $2 \times 3$ contingency table was applied, "'The novel SNP $(-1541 \mathrm{C}>\mathrm{T}$; agcacttgggaggc c/t gaggcgggtggatcgc) and variation sites (-1292_-1289delTAAA; ctccatctcaaaaaaataaataaa taaa/del taaaaataaaataaaataaaatgagaaattgattat and -1282_-1278dupTAAAA; ctcaaaaaaataaataaataaataaaaataaaataaaa taaaa/dup tgagaaattgattatgatctc) identified in this study. 


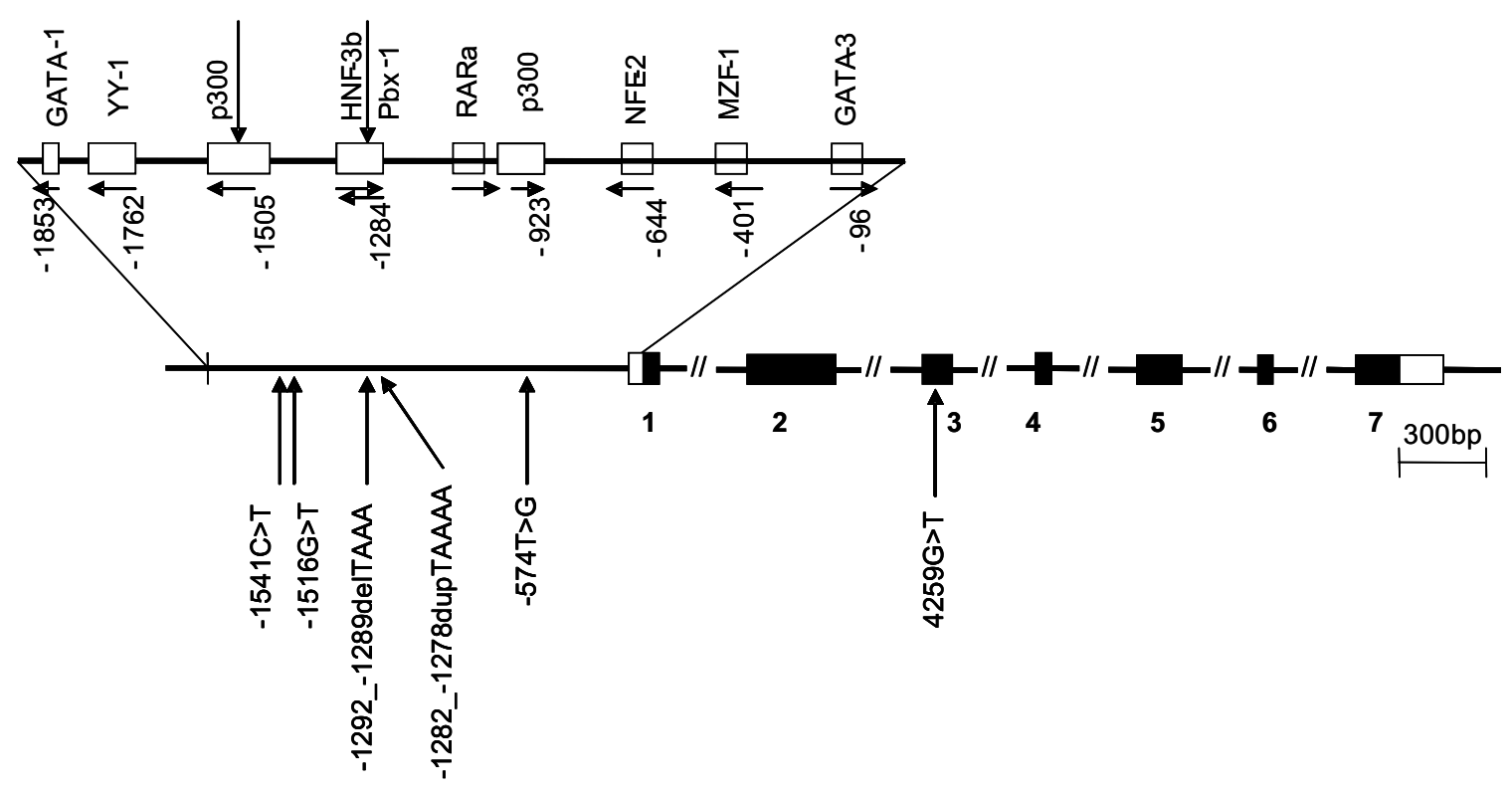

Figure 1. Locations of each single nucleotide polymorphisms (SNPs) and variation sites in Tim-3. Coding exons are marked by black blocks and 5'- and $3^{\prime}$-UTR by white blocks. The positions of SNPs were calculated from the translation start site. Putative transcription factor sites are searched at www.cbrc.jp/research/db/TFSEARCH.html. The reference sequence is based on human chromosome 5 clone CTB-120L21.

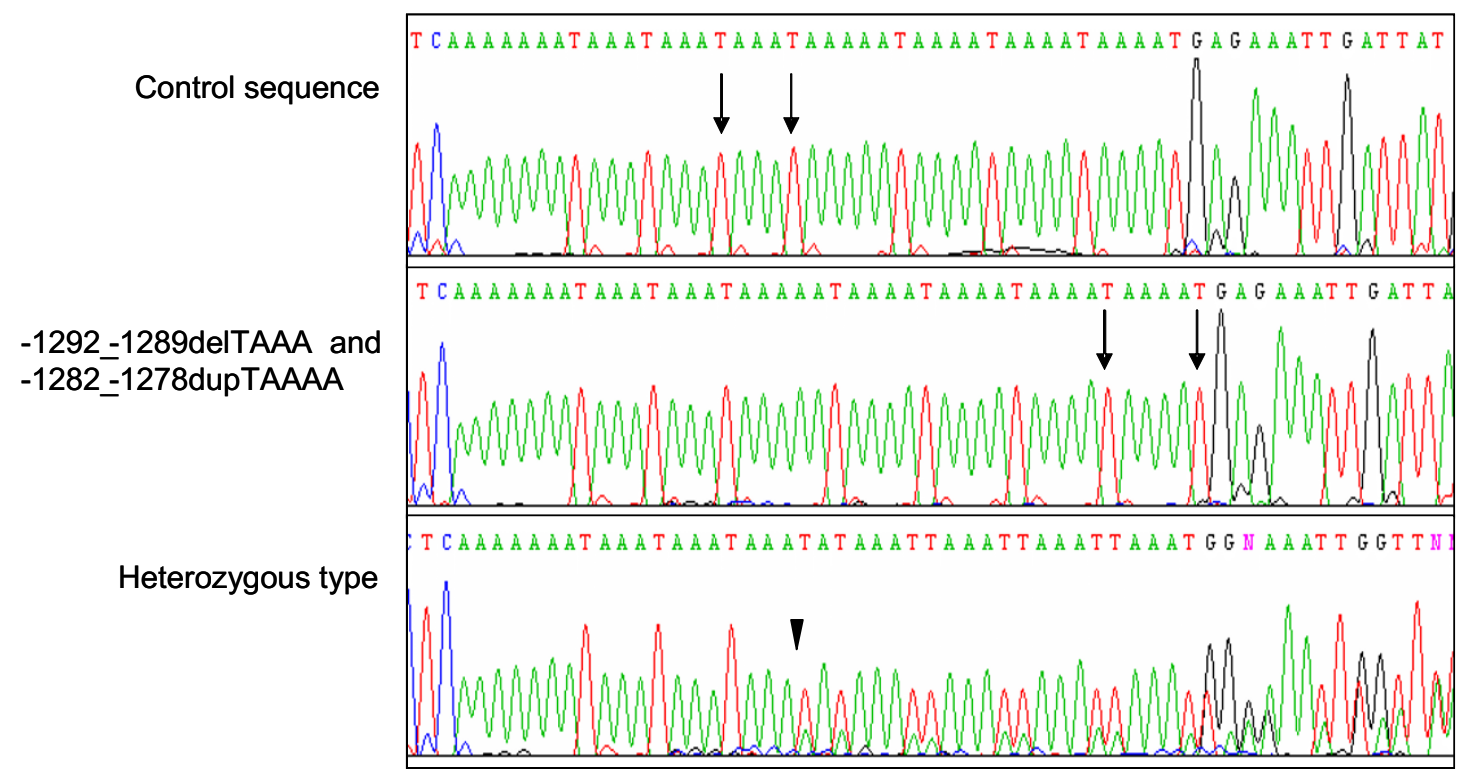

Figure 2. DNA sequence chromatograms of -1292_-1289delTAAA and -1282_-1278dupTAAAA variation sites of Tim-3 promoter region. PCR products purified by PCR purification kit (Millipore, USA) were used as the template DNA for cycle sequencing. Sequence analysis was performed by BigDye Terminator cycle sequencing using an ABI 3100 Prism Automated DNA sequencer (Applied Biosystems). The arrows indicate variation sites.

tion site, -1292_-1289delTAAA was combined with -1282 -1278dupTAAAA variation (Figure 2). These variations were found the all of experimental samples $(n=74)$ for this study and located at the putative transcriptional binding site of HNF-3b, which is hepatic nuclear factor 3 beta (Figure 1). The homo- geneous variation occurred to high frequency $(75.7 \%)$, while -1292_-1289delTAAA and -1282_-1278dupTAAAA variations in some cases concurrently existed as shown in Figure 2. The $P$ values of each SNPs or variation sites were not significant between controls and asthma or allergic rhinitis patients (Table 2). 
Mclntire et al. identified major polymorphisms in TIM-3 that was strongly associated with Th1/Th2 differentiation and the expression of airway hyperreactivity (AHR) in congenic mice (Mclntire et al., 2001). They compared the TIM-3 amino acid sequence predicted from the BALB/C that develop Th2 biased immune responses with enhanced $A H R$ and $H B A$ (DBA/2) that develop reduced IL-4 responses, and found amino acid differences at seven positions. In this study, we identified only one polymorphism from the coding region of human Tim-3 gene. This result suggests that the polymorphic sites of Tim-3 gene in human differ from that of mice. GG genotype at 4259G $>T$ (rs1036199) was changed to TT in all genomic DNA of both healthy controls and asthma patients (Table 2). Although it is not yet known whether these newly described polymorphisms are associated with some functions, it will be important in future studies to determine whether or not this polymorphism affects the levels of TIM-3 production. It would also be interesting to analyze the allele frequency of these variances with large sample size in patients with autoimmune and allergic diseases.

\section{Acknowledgement}

This work was supported by a grant from the Korea Health 21 R\&D Project by Ministry of Health and Welfare (01-PJ3-PG6-01GN09-003).

\section{References}

Feigelstock D, Thompson P, Mattoo P, Zhang Y, Kaplan GG. The human homolog of HAVcr-1 codes for a hepatitis A virus cellular receptor. J Virol 1998;72:6621-8

Ho IC, Glimcher LH. Transcription: Tantalizing Times for T Cells. Cell 2002;109:S109-20

Hofstra CL, Van Ark I, Hofman G, Kool M, Nijkamp FP, Van
Oosterhout AJ. Prevention of Th2-like cell responses by coadministration of $\mathrm{IL}-12$ and $\mathrm{IL}-18$ is associated with inhibition of antigen-induced airway hyperresponsiveness, eosinophilia, and serum IgE levels. J Immunol 1998;161: 5054-60

Kim YJ, Lee HS, Im JP, Min BH, Kim HD, Jeong JB, Yoon JH, Kim CY, Kim MS, Kim JY, Jung JH, Kim LH, Park BL, Shin HD. Association of transforming growth factor- $\beta 1$ gene polymorphisms with a hepatocellular carcinoma risk in patients with chronic hepatitis B virus infection. Exp Mol Med 2003;35:196-202

Kumanogoh A, Marukawa S, Suzuki K, Takegahara N, Watanabe C, Ch'ng E, Ishida I, Fujimura H, Sakoda S, Yoshida K, Kikutani H. Class IV semaphorin Sema4A enhances T-cell activation and interacts with Tim-2. Nature 2002;419:629-33

Lack G, Renz H, Saloga J, Bradley KL, Loader J, Leung DY, Larsen G, Gelfand EW. Nebulized but not parenteral IFN-g decreases $\operatorname{lgE}$ production and normalizes airways function in a murine model of allergen sensitization. J Immunol 1994;152:2546-54

McIntire JJ, Umetsu SE, Akbari O, Potter M, Kuchroo VK, Barsh GS, Freeman GJ, Umetsu DT, DeKruyff RH. Identification of Tapr (an airway hyperreactivity regulatory locus) and the linked Tim gene family. Nat Immunol 2001;2:110916

Monney L, Sabatos CA, Gaglia JL, Ryu A, Waldner H, Chernova T, Manning S, Greenfield EA, Coyle AJ, Sobel RA, Freeman GJ, Kuchroo VK. Th1-specific cell surface protein Tim-3 regulates macrophage activation and severity of an autoimmune disease. Nature 2002;415:536-41

Nicholson LB, Greer JM, Sobel RA, Lees M B, Kuchroo VK. An altered peptide ligand mediates immune deviation and prevents autoimmune encephalomyelitis. Immunity 1995;3: 397-405

O'Garra A. Commit ye helpers. Nature 2000;404:719-20.

Umetsu DT, Mclntire JJ, Akbari O, Macaubas C, DeKruyff $\mathrm{RH}$. Asthma: an epidemic of dysregulated immunity. Nat Immunol 2002;3:715-20 